\title{
The Acceptance Status of Traditional Moral Culture in Colleges and Universities Using Convolutional Neural Network
}

\author{
Bingduan Liu (i) \\ School of Art and Design, Wuhan Institute of Technology, Wuhan 430205, China \\ Correspondence should be addressed to Bingduan Liu; studytochina@wit.edu.cn
}

Received 10 December 2021; Accepted 27 December 2021; Published 24 February 2022

Academic Editor: Muhammad Usman

Copyright (C 2022 Bingduan Liu. This is an open access article distributed under the Creative Commons Attribution License, which permits unrestricted use, distribution, and reproduction in any medium, provided the original work is properly cited.

\begin{abstract}
In today's world, competition among countries is not just a contest of hard force, but also a contest of soft power. China has a fivethousand-year-old history and civilization, with a strong traditional moral culture at its core. The university stage is an important time for college students to develop their worldview, outlook on life, and values. In this context, excellent traditional and moral culture education plays an important role and significance in their development. College students are the country's future builders and an important force to inherit China's excellent moral culture. The rapid development of the modern Internet era has made the channels for college students to understand and accept the cultures of various countries more and more diverse and convenient. Actively borrowing their essence can help promote the development of Chinese culture, but we must also pay attention to active defense and resistance against the dregs of culture. This paper discusses the current situation and problems of the acceptance status of excellent traditional moral culture among college students and analyses the factors that restrict the acceptance of excellent traditional moral culture among college students to conduct a large-scale study on the acceptance status of traditional moral culture in colleges and universities. A model based on convolutional neural networks is suggested for predicting college students' embrace of traditional moral values. By learning and describing their activities and preferences on the Internet, the model assesses and creates college students' acceptance status for conventional moral culture. Extensive tests indicate that the suggested technique can successfully assess college students' acceptance status for traditional moral culture, and the model's superior performance is supported by test results.
\end{abstract}

\section{Introduction}

With the increasingly harsh rivalry among nations' comprehensive national strength, the competition of countries' soft power is receiving more and more attention and importance in today's peaceful development. As the motherland's future leaders, college students' attitudes toward and acceptance of outstanding traditional moral culture play a critical role in preserving history and culture and actively advancing excellent moral culture. In this new period of fast economic globalization, China's great traditional moral culture is now confronted with a challenging foreign and local environment. College students should actively accept Chinese excellent traditional moral culture and, in light of China's current national circumstances, grasp the international development trend to better transform and carry forward Chinese excellent traditional moral culture and promote China's soft power development [1].

With the advent of the Internet era, college students are exposed to a plethora of international and domestic cultural information, including many foreign excellent and dreary cultures, which has a direct impact on the moral and cultural outlook of college students and naturally leads to clashes with traditional Chinese culture [2]. The author plotted the hotness trend of the search phrase Chinese traditional moral culture in Google from 2004 to 2021, as shown in Figure 1, and we can see that the hotness trend is steadily diminishing, suggesting that the Internet's attention to Chinese traditional moral culture is constantly declining. College students bear the important responsibility of inheriting excellent traditional moral culture as the driving force of future socialist modernization construction, and their attitude toward 


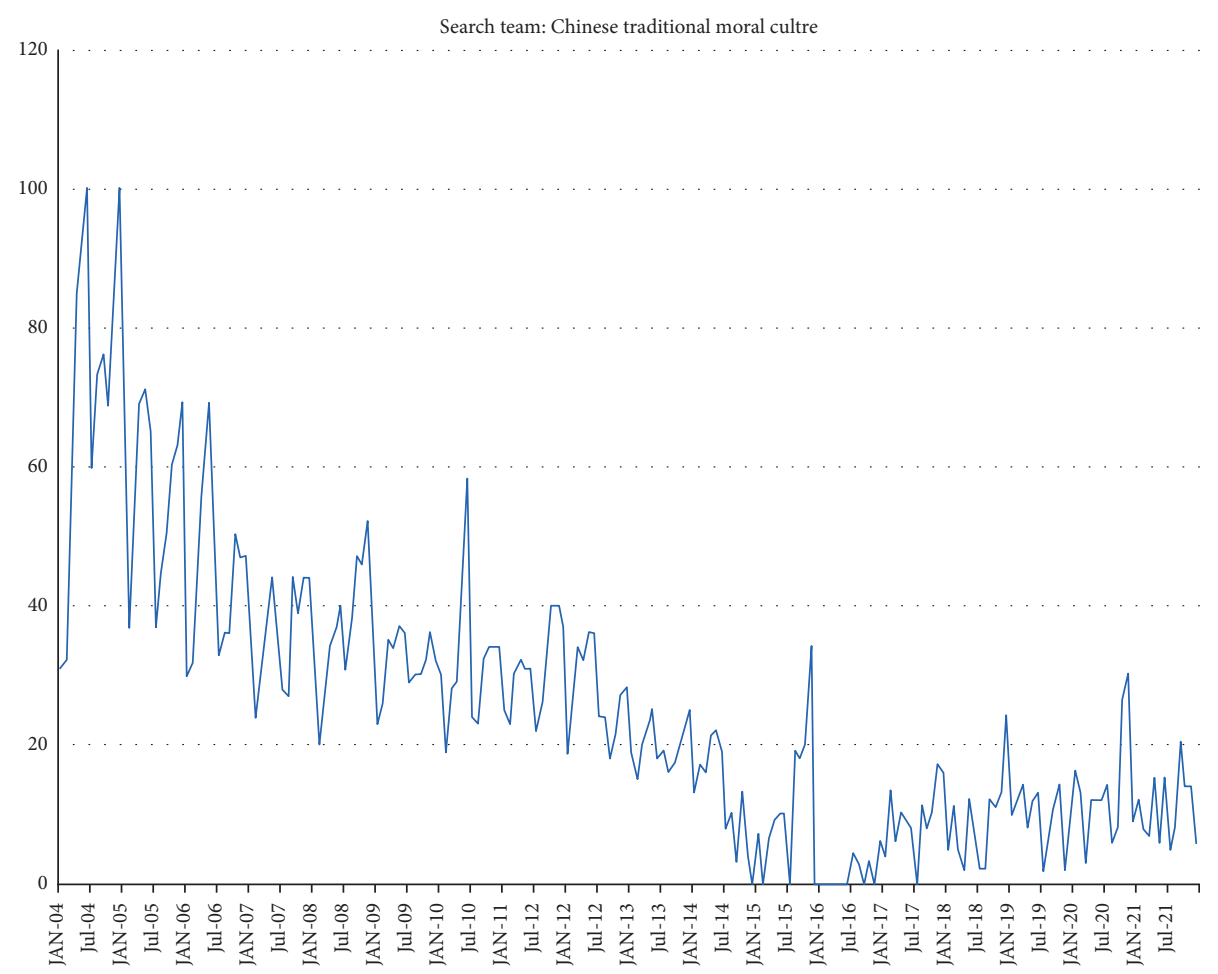

FIgURE 1: Google hot trends chart.

accepting excellent traditional moral culture is extremely important and should be given more and more attention and concern. The acceptance state of outstanding traditional moral culture among college students is the study object of this work, which examines the acceptance state, degree, and routes of current college students to excellent traditional moral culture. The purpose of this paper is to examine the acceptance status [3], degree, and channels of excellent traditional Chinese moral culture among contemporary college students to carry forward excellent traditional Chinese moral culture, build an advanced culture with the times, and strengthen China's soft power construction. The university stage is critical for the formation and improvement of one's worldview, outlook on life, and values, and in an era when the world is moving toward a knowledge-based economy and a technology-based national strategy, university students are the country's hope for prosperity and strength. Acceptance and inheritance of good traditional moral culture by college students is highly regarded by people from all walks of life, and it is critical to assemble the Chinese nation's solid will and renew the great national spirit to realize the Chinese nation's great rejuvenation. Acceptance of outstanding traditional moral culture by college students is also important for improving their overall strength and cultural literacy [4]. Confucianism, Taoism, and Buddhism, which are at the heart of China's great traditional moral culture, may assist college students in overcoming the challenges and problems they face in everyday life and their studies, as well as nurturing high connotations in college students. As a result, the purpose of this article is to create awareness of the need of adopting and cultivating outstanding traditional Chinese moral culture among college students, so that they may comprehend the relevance of doing so in their future studies, lives, and job [5].

Traditional moral culture education for college students is an education that aids in the development of outstanding moral character among college students. The fast growth of the Internet, particularly social networking sites, presents both possibilities and problems for conventional moral culture teaching for college students. Colleges and universities may take advantage of the central role of social networks in college students' everyday lives to develop a new model of the traditional moral culture education and actively guide the development of excellent traditional moral and cultural character in college students. One of the most important characteristics of high-quality talents is a noble character, which allows for a wide spectrum of synergistic growth. Noble personal character and values are beneficial to people's peaceful contact with others in the process of selfcontrol, while also encouraging harmonious social growth, in social activities. Colleges and universities should make extensive use of social media to encourage the formation of traditional moral culture among students [6]. It gives college students a suitable teaching platform for traditional moral culture instruction. Traditional moral culture education in schools is limited to the teaching of textbook contents, and students mechanically complete learning tasks. As a result of the negative effects of exam-oriented education, colleges and universities place a greater emphasis on instilling theoretical knowledge of traditional moral culture in students, which to some extent inhibits students' interest, reduces teaching efficiency and results, and is not conducive to student play. Social networks, on the other hand, ignore these flaws and 
place a greater focus on the central role of students, offering a platform for college students to learn more about conventional moral culture's ideological and theoretical knowledge as well as new thinking patterns [7]. It provides a variety of educational tools for college students' traditional moral culture instruction. The diverse information distribution contents of social networks contribute to the enrichment of conventional moral culture instructional resources. Educators can help college students' ideologies become less confined by providing them with relevant knowledge and news, as well as assisting them in taking a step back to consider their benefits and disadvantages from a higher perspective. At the same time, college students may pick their learning materials at any time and from any location, enhancing their creative awareness and desire to study.

Social networks are more significant in the everyday lives of college students in the contemporary era of social networks, and they have a profound impact on their consciousness and conduct. As a result, the study of traditional moral culture acceptance in colleges and universities in a social network setting provides the foundation for providing traditional moral culture education to college students. However, in the social network setting, college students' behaviors and preferences are abstract and difficult to quantify, and their acceptance status of conventional moral culture is similarly oblique and implicit. This research offers a model based on convolutional neural networks to correctly assess the acceptance status of traditional moral culture in colleges and universities. The model first obtains college students' preferences in social networks and characterizes them as digital features, then extracts high-level semantic information from digital features using a convolutional neural network, and finally uses a classifier to discriminate college students' acceptance status for traditional moral culture. Extensive studies reveal that the suggested technique properly identifies college students' acceptance status for traditional moral culture, laying the groundwork for furthering traditional moral culture instruction in colleges and universities in the social network environment. Main contributions of this research work are listed below:

(i) Due to several factors such as social evolution and cultural conflicts, school reform and teacher quality, family moral education environment, and college students' self-restraint ability, this work finds that the existing problems primarily include a lack of awareness among college students themselves, an imperfect education mechanism, and a lack of practical activities.

(ii) Based on their actions and preferences in social networks, the suggested model predicts college students' acceptance status of Chinese good traditional moral culture.

(iii) Finally, studies in the test set demonstrate the validity and accuracy of the proposed model by constructing a dataset with actual questionnaires, laying the groundwork for future instruction on Chinese superb traditional moral culture for college students' groups.
The rest of the paper is organized as follows: Section 2 explains the related work, Section 3 presents the methodology for the proposed model, Section 4 explains experimentation and evaluation results that the author has obtained during work, and finally, the author concluded the work in separate section as Section 5 .

\section{Related Work}

In this section, the author discusses works related to the research topic. Here social networks and challenges posed by social networks and convolutional neural networks are first explained.

2.1. Social Networks. Simply said, social networks are webs of social interactions and personal connections. Consider how you met your pals and how you got to know them. Perhaps you met them in primary school, or perhaps you met them via a pastime or through your church. In either case, you were exposed to social networks, which entail meeting new people in a social setting and forming strong personal relationships over time. While social networking concentrates upon allowing like-minded people to communicate with one another through websites and web-based apps, social networking sites include Facebook, MySpace, Twitter, and LinkedIn. The word "social network" has a fairly broad definition since it is still a relatively new technology that is prone to fast development. As indicated in Figure 2, there are three main types of social networks, and an integrated social business strategy uses all three to increase communications with various groups of people.

(i) Public social networks: these networks, like Facebook and Twitter, are useful for connecting with consumers and prospects. These networks solve simple issues.

(ii) Social extranets: these networks include customer communities, which allow for deeper engagement and cooperation with customers, as well as private business-to-business networks, which allow for communication with partners and B2B customers. In these networks more focus is on engagement.

(iii) Employee networks: these are used for internal corporate communication. Most of these networks share knowledge and response better that above.

2.2. The Challenges Posed by Social Networks. For China's education model, the emergence and development of social networks have also promoted the reform and innovation of China's traditional education model to a certain extent and played a positive role in promoting the construction of China's advanced culture [8]. The form of education in the era of social network is no longer restricted by geography, the circle of friends can be extended to all corners of the world, and college students can use their virtual identities to communicate and study with the outside world, confide their inner feelings without any worries, and relieve the 


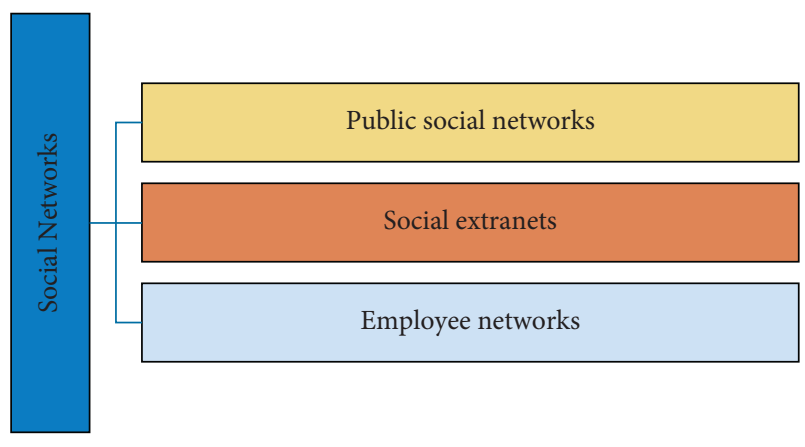

Figure 2: Types of social networks.

pressure brought by their study life [9]. This enriches the lives of college students and satisfies their spiritual needs. The social network environment focuses on the development of personal ability and ignores the beneficial effect of personal cultivation and traditional moral culture on their own progress. Under the influence of this negative concept, college students mistakenly believe that the development of society needs only people with higher personal expertise and skills, which leads college students to neglect the formation of traditional moral culture and lack of binding force on their personal behavior in the process of learning professional knowledge. Therefore, students set their main goals on professional knowledge, computer knowledge, foreign languages, etc. and participate in various club activities to improve their ability to do things and social skills, but they are not motivated to study the content of traditional moral and cultural education. This leads to some college students' rude speech and behavior, noncompliance with public order, and poor self-restraint ability. At the same time, because college students lack certain social experience, have little sense of right and wrong, and do not have enough immunity to the external environment, they are easily influenced by the bad social values, and their ideology and behavior consciousness deviate from the moral standards. As the main group of social media and social networking sites such as microblogs and WeChat, college students are easily influenced by some bad information in social networks, thus distorting their not yet fully mature outlook on life, values, and worldview [10]. For example, the most common kind of negative energy circulating on the network nowadays, namely, the show of wealth, the excessive worship, and enjoyment of material enjoyment, can easily influence the values of college students and focus one-sidedly on the psychological satisfaction brought by material conditions, which leads college students to excessively pursue material benefits in the learning process.

In addition to affecting the learning effect, more importantly, it is not conducive to the formation of good personal cultivation of college students. It is not conducive to the formation of good traditional moral culture of college students. The initial formation of people's traditional moral concept comes from parental education and family environment influence, which is the basis for further improvement of moral concepts after entering society. However, contemporary college students generally [11] have the phenomenon of weak moral concepts in their families. For example, college students fall in love, due to the characteristics of the wide coverage of social networks, the concept of love of college students is influenced by the Western open ideology, and deviations in the concept of love and morality appear. To satisfy their material life or fill their inner emptiness, some students fall in love with an extremely irresponsible attitude, and what is more outrageous is that some students decide the love object through games, do not take feelings as the starting point, choose the love object at will or change the love object frequently, are not bound by ethics and morality, and show their personal ability through love. The spread of bad social concepts in social networks largely affects the behavioral norms and social ethics of college students and is not conducive to the formation of good traditional moral concepts of college students [12]. It is not conducive to the formation of responsible social and public consciousness of college students.

2.3. Convolutional Neural Networks. The convolutional neural network $(\mathrm{CNN})$ is a deep learning architecture inspired by the visual system. Hubel and Wiesel discovered it in 1962 while studying the primary visual cortex of cats. Receptive fields are tiny subregions of the visual field that are responsive to cells in the cortex [23]. These cells are responsible for detecting light in the receptive fields. In 1980, Fukushima introduced Neocognitron, which was based on Hubel and Wiesel's work and was the first computer-simulable model. This Neocognitron is regarded as the forerunner of CNNs, and it was based on the hierarchical structure of neurons for image processing. By creating an artificial neural network with a multilayer termed LeNet-5, the author of [24] laid the groundwork for CNNs. The backpropagation technique was used to train this artificial neural network, which was employed to do handwritten digit categorization. It was possible to recognize patterns from raw pixels after training with this approach. Despite its numerous advantages, LeNet- 5 has not been effective in handling complicated issues such as video categorization.

Artificial neural network (ANN) is a hot research topic that emerged in the field of artificial intelligence in the 1980s [13]. It abstracts the neuronal network of the human brain from the perspective of information processing, builds some simple models, and forms different networks according to different connections. It is often directly referred to as a neural network or neural-like network in engineering and academic circles. A neural network is an operational model consisting of a large number of nodes (or neurons) connected. Each node represents a specific output function, called the activation function- $n$. Each connection between two nodes represents a weighted value of the signal passing through that connection, called weight, which is equivalent to the memory of an artificial neural network. The output of the network varies depending on the connection method, weight, and excitation function of the network. The network itself is usually an approximation of some algorithm or function in nature, or it may be an expression of a logical strategy. In the last decade or so, the 
research work of artificial neural networks has been deepening and has made great progress, and it has successfully solved many practical problems that are difficult to be solved by modern computers in the fields of pattern recognition, intelligent robotics, automatic control, prediction estimation, biology, medicine, economy, etc., and has shown good intelligent properties. With the rapid development of neural networks, their definitions have also emerged. As early as 1989, YannLeCun and his colleagues published the results of convolution neural networks (CNN) [14], a deep neural network with a convolutional structure, usually with at least two nonlinear trainable convolutional layers and two nonlinear fixed convolutional layers (also known as pooling). The structure of CNNs is inspired by the famous Hubel-Wiesel model of biological vision, especially to simulate the behavior of Simple Cell and Complex Cell in layers V1 and V2 of the visual cortex. The evolutionary path of CNN can be summarized into 4 paths: (1) deeper network; (2) enhanced convolutional mode, and fusion of the above two ideas; (3) from classification to detection; and (4) addition of new functional modules. More and more $\mathrm{CNN}$ models are now evolving step by step from giant networks to lightweight networks, and the model accuracy is getting higher and higher. The focus of the industry has now shifted from accuracy improvement (because they are all already high) to a balance of speed and accuracy [14], all wanting the models to be fast and accurate. Therefore, from the original AlexNet [15] and VGG-Net [16] to the smaller Inception and ResNet [17] series and then to the current MobileNet [18] and ShuffleNet [19] that can be ported to mobile, we can see some trends as follows. Convolutional kernels: (1) large convolutional kernels are replaced by multiple small convolutional kernels; (2) single-size convolutional kernels are replaced by multisize convolutional kernels; (3) circumscribed shape convolutional kernels tend to use deformable convolutional kernels; (4) $1 \times 1$ convolutional kernels are used. Convolutional layer channels: (1) standard convolution is replaced by depth-wise convolution; (2) grouped convolution is used; (3) channel shuffle is used before grouped convolution; (4) channel weighting is calculated. For convolutional layer connection: (1) use skip connection to make the model deeper; (2) densely connect so that each layer incorporates the feature outputs of the other layers. Convolutional neural networks are still evolving and innovating, but each advancement is an improvement and innovation based on the previous neural network structure, which makes up for the shortcomings of the existing network. After AlexNet [15], there are two major directions of innovation, one is the improvement of the network structure and the other is the improvement of the network depth. However, the current neural network has a huge number of parameters, and the requirement of computational power is still very high. How to choose the appropriate hyperparameters, such as learning rate, kernel size of convolutional filtering, number of layers, etc., requires a lot of technology and experience, which is also the future development direction of convolutional neural networks and needs more in-depth research.

\section{Method}

In this section, the author explains the methodology that was adopted for the proposed research work.

3.1. Data Collection. The study of different college students' acceptance status of traditional moral culture in colleges and universities using social media data is mainly conducted by text data mining techniques based on convolutional neural networks. First, based on the Weibo and WeChat accounts raised by volunteers, crawler technology is used to obtain textual information related to Weibo and WeChat users, which is used to build a database and train a model.

In recent years, the emergence and development of microblog social platforms have led to a dramatic change in the way people use the Internet, transforming from simple web browsing and information searching to information mining, communication, and sharing. Especially since 2010, microblogs have rapidly penetrated among Internet users by virtue of their platform's openness, terminal scalability, and content simplicity and gradually developed into an important social media, becoming an important medium for college students to obtain real-time news, social sharing, and social participation. After the processing work of deduplicating and summarizing microblog information by date, the content of microblog texts posted, commented, retweeted, and liked by a certain college student is the core data of this study, as shown in Table 1.

At present, millions of WeChat public resources are accumulating information and quality content, which has become the main way for a considerable number of users to obtain information. However, due to the limitations of WeChat's social properties and the fact that it is not the same as traditional web pages that link to each other through hyperlinks, these massive quality resources have not been effectively released and have existed in an "island" in the current Chinese Internet world. The core indicators of this study are the number of tweeted articles in the public number subscribed by a college student, the text posted in the circle of friends, and the collected articles, after summarizing the information of WeChat. For these core indicators, after finishing the calculation, this paper designs the main basis of WeChat pushed articles, the content posted by users, and the text content collected by users, as shown in Table 2.

3.2. Model Architecture. In this section, the author explains architecture of the model. The flowchart of traditional moral culture acceptance state model in colleges and universities based on convolutional neural network is shown in Figure 3.

In this paper, the test data obtained from microblogs and WeChat are cleaned, truncated, and sequence-labelled, and the preprocessed data are input into a BERT model [20], and its output is connected to a convolutional neural network model for identifying the acceptance status of traditional moral culture by college students. The model used in this paper contains two structures: the BERT model and the convolutional neural network model [21]. The two models 
TABLE 1: Weibo indicators.

\begin{tabular}{lc}
\hline $\begin{array}{l}\text { Indicator } \\
\text { name }\end{array}$ & Description \\
\hline Post content & Content of Weibo posted by each university \\
student \\
$\begin{array}{l}\text { Comments } \\
\text { Retweets }\end{array}$ & Content of Weibo posts per student \\
Likes & Retweets by each student \\
\hline
\end{tabular}

TABLE 2: WeChat indicators.

\begin{tabular}{lc}
\hline Indicator name & \multicolumn{1}{c}{ Description } \\
\hline Push content & Content pushed to users by WeChat \\
Publish content & Content posted by users on WeChat \\
User favorites & Content collected by users on WeChat \\
\hline
\end{tabular}

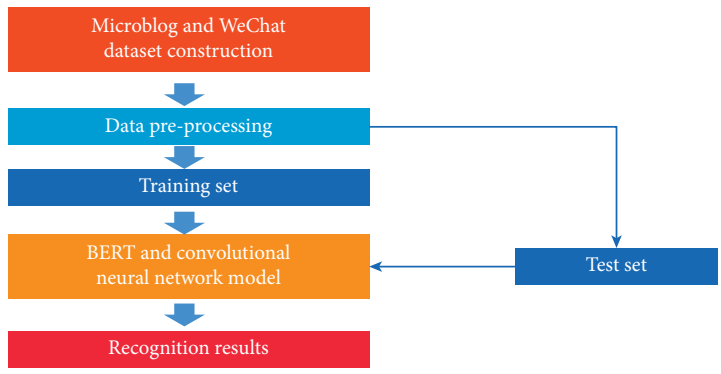

FIgURE 3: Flowchart.

are connected hierarchically. The overall structure of the model is shown in Figure 4.

3.3. BERT Model. The model structure of BERT is a multilayer bidirectional converter structure, and unlike other recent language representation models, BERT [21] aims to pretrain deep bidirectional representations by jointly regulating the context in all layers. In the encoding process of the converter, the data are first passed through the self-attentive module to obtain a weighted feature vector:

$$
Z=\operatorname{Attention}(Q, K, V)=\operatorname{soft} \max \left(\frac{Q K^{T}}{\sqrt{d_{k}}}\right) V,
$$

where $Q, K$, and $V$ are obtained from 3 embedding vectors, respectively. The feature vector $Z$ is passed through the feedforward neural network to obtain the word encoding.

$$
\operatorname{FFN}(Z)=\max \left(0, Z W_{1}+b_{1}\right) W_{2}+b_{2} .
$$

The input of BERT is composed of three parts: (1) character embedding represents the embedding of the current word; (2) paragraph embedding represents the index embedding of the sentence where the current word is located; (3) location embedding represents the index embedding of the location of the current word. The input is "I won the lottery today, I'm buying dinner," and the output of BERT is a vector representation of the input words with the full-text semantic information, which is a pretraining model using the mask language model and the next sentence prediction model. Masked language model: $15 \%$ of the characters in the input vector are masked out randomly. When training the model, a vector is fed into the model several times for parameter learning, and instead of masking out the words each time, the words to be masked out are replaced with "[Mask]" in $80 \%$ of the time, with any other words in $10 \%$ of the time, and with the original marker.

3.4. Convolutional Neural Networks. The word vector with global features output from BERT is used as the input of the convolutional neural network, which is different from image convolution, which uses a two-dimensional vector, and text convolution, which uses a one-dimensional vector. This paper adopts the structural model of a one-dimensional convolutional layer with a maximum pooling layer classification layer and adds a discard layer to prevent overfitting and overparameterization during the training of the model [22]. After preprocessing a text sequence of length $n$, the feature mapping of each word is obtained by encoding the BERT pretraining model as follows:

$$
e_{x_{i}}=E\left[x_{i}\right]
$$

where $x_{i}$ denotes the word vector corresponding to character $i$ at position $i$, and $E\left[x_{i}\right]$ denotes the dense vector of word vectors trained in the model. The feature mapping of the sequence is denoted as

$$
e_{i: n}=e_{x_{1}} \oplus e_{x_{2}} \oplus \cdots \oplus e_{x_{n}}
$$

$e_{x_{1}}$ denotes the vector that is in the first position in the sequence and $*$ denotes the concatenation operator. The word vector is passed through a one-dimensional convolutional layer to obtain the feature mapping $c_{i}$.

$$
c_{i}=f\left(w_{i}^{*} e_{x_{i}}+b\right),
$$

where $w_{i}$ denotes the weight and $b$ denotes the bias. The feature mapping is expressed as

$$
c=\left[c_{1}, c_{2}, \ldots, c_{n}\right] .
$$

After maximizing the pooling layer to obtain the feature vector:

$$
\widehat{c}=\max (c) .
$$

The classification results after the fully connected layer:

$$
P\left(y \mid x_{1}, x_{2}, \ldots, x_{n}\right)=\operatorname{soft} \max (\widehat{c}) .
$$

\section{Experimentation and Evaluation}

4.1. Dataset. In order to construct the dataset, the author first designed the Questionnaire on the Acceptance Status of Excellent Traditional Moral Culture among College Students. This questionnaire is divided into three parts: objective information, subjective questions, and social network information, involving contemporary college students' attitudes toward, mastery of, and opinions and suggestions on excellent traditional Chinese moral culture, and mainly includes the 


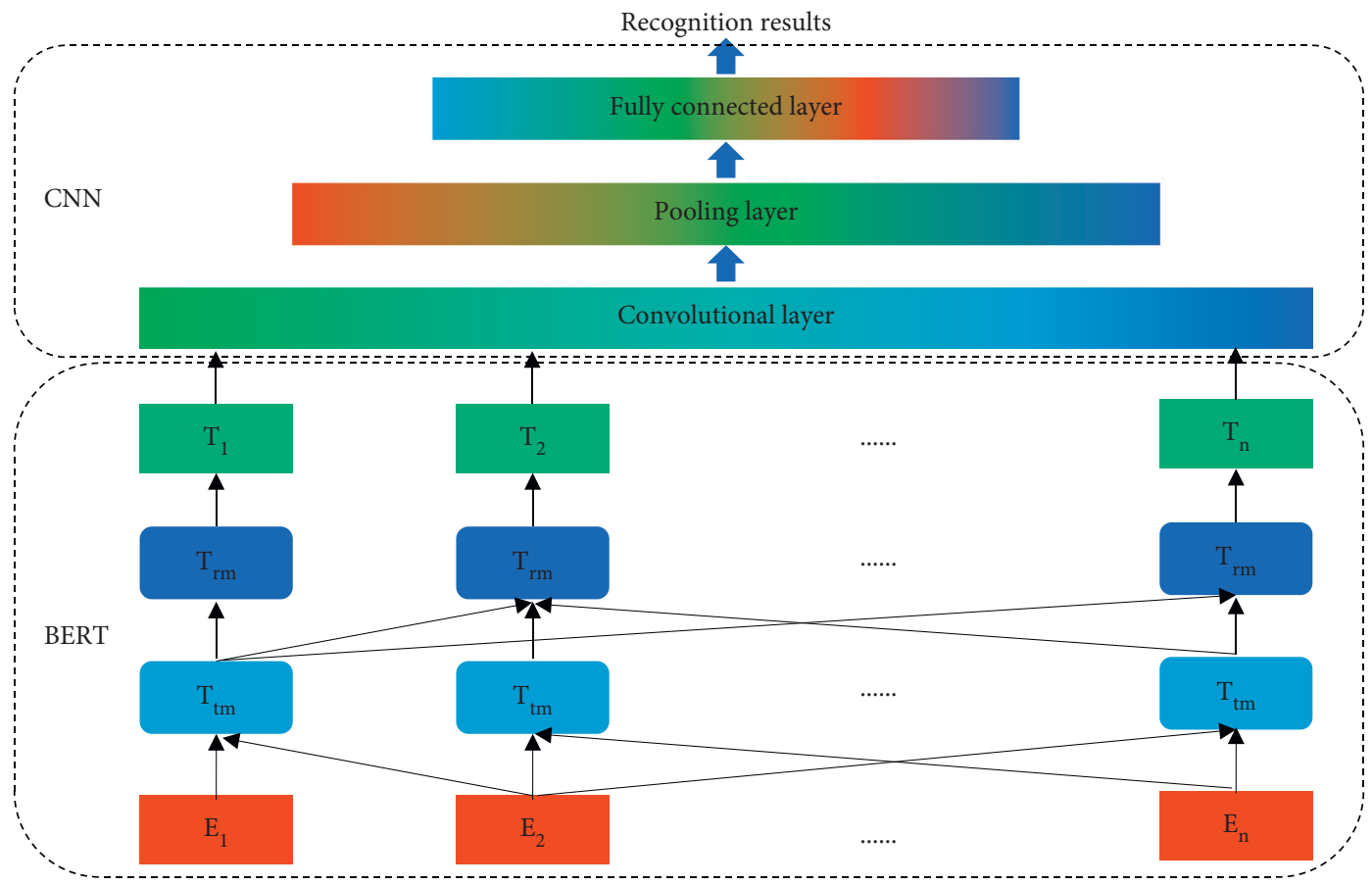

Figure 4: Model architecture.

following types of questions: (1) Overall acceptance status of excellent traditional moral culture among college students, denoted as C1. (2) Acceptance status of political morality such as serving the country with fidelity and national temperament, denoted as C2. (3) The acceptance status of righteousness and profit cultivation, such as self-restraint and righteousness, is expressed as C3. (4) The acceptance status of social morality, such as honesty and knowledge of reward, modesty, and benevolence, is expressed as C4. (5) The acceptance status of human morality, such as family harmony and filial piety, is expressed as C5. (6) The acceptance status of enterprising spirit, such as pragmatism and self-improvement, is expressed as C6. The questions are closely matched with the life of college students and have high practicality. The objective information and subjective questions constitute the labels of each college student's acceptance status of traditional moral culture in colleges and universities, and the social network information constitutes the sample data of college students' acceptance status of traditional moral culture in colleges and universities. The social network information was crawled using crawler technology to crawl relevant text data. The survey questionnaires were distributed to undergraduate and graduate students in several universities, with a total of 500 volunteers. To ensure that the questionnaires could be effectively collected, a combination of field and correspondence distribution was used between May 30, 2021, and July 10, 2021, and a stratified sampling method was used to distribute questionnaires to several school students (see Table 3), and a total of 500 questionnaires were distributed and 473 were collected, removing invalid questionnaires and making a total of 462 valid questionnaires. The dataset was divided into 312 training samples, 50 validation samples, and 100 test samples.
4.2. Experimental Setup. The proposed method operating system is Linux 16.0, programming environment is python3.6 pytorch-gpu, GPU is GXT 2080TI, memory is 64G, and CPU is Intel (R) Core (TM) i5-9300H $2.4 \mathrm{GHz}$. the model optimization method uses Adam gradient descent and cross entropy loss function method, the learning rate is set to 0.0004 by debugging, the batch size is set to 32 , and the discard layer parameter is set to 0.4 in order to avoid overfitting and overparameterization of the model during training.

4.3. Experimental Results. The loss curves during the model iterations are shown in Figures 5 and 6. As seen in Figure 6, the model is trained for 5 times (300 iterations) when the loss curve plateaus and the classification effect is optimal. The model's test accuracy increases with the number of trainings with the same number of test sets as shown in Figure 7. Figure 7 shows that the model accuracy gradually increases with the increase of training data, and it is difficult to achieve the desired model accuracy with a small number of datasets, and the accuracy of the model needs a large amount of data support.

4.3.1. C1 Category Acceptance Status. The survey results show that college students' overall acceptance of good traditional morality is average, and their overall willingness to accept is generally not strong, while their knowledge mastery is good, but they fail to reach the most ideal acceptance state in terms of interpretation, screening, and practice. When answering the question "What is your overall evaluation of your own acceptance of good traditional moral culture," as 
TABle 3: Survey statistics table.

\begin{tabular}{lccccc}
\hline Student type & Number of distribution & Number of recycling & Recycling rate (\%) & Effective number & Effective recycling rate (\%) \\
\hline Undergraduates & 350 & 329 & 94.0 & 322 & 97.8 \\
Graduate students & 150 & 144 & 96.0 & 140 & 97.2 \\
\hline
\end{tabular}

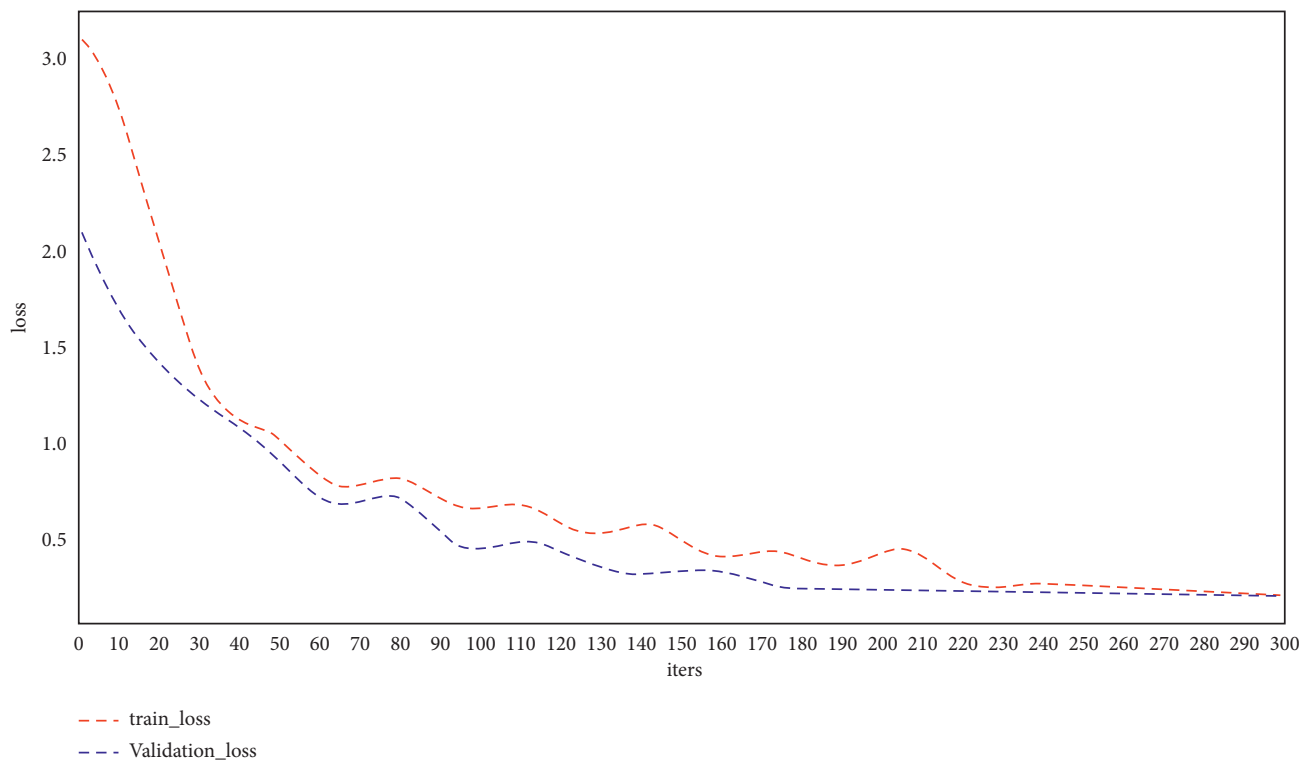

FIgURE 5: Training and validation loss trend graphs.

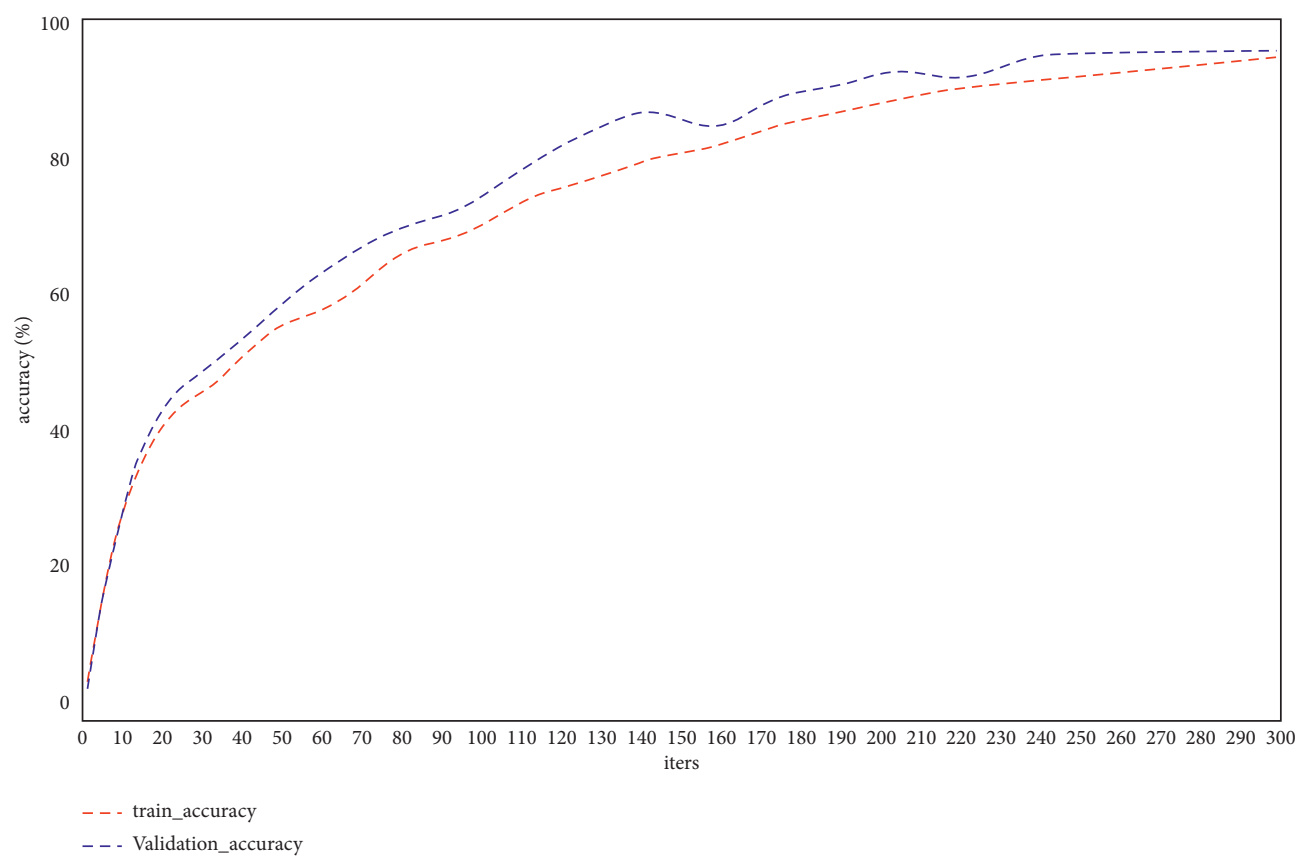

FIgURE 6: Training and validation accuracy trend graph.

many as $43 \%$ of the students chose "average," $8 \%$ chose "poor," $39 \%$ chose "good," and only $10 \%$ of college students think their own acceptance of good traditional culture is excellent. The overall acceptance result predicted by the model is almost the same as the actual one.
4.3.2. C2 Category Acceptance Status. From the data in Table 4 , it can be seen that $71 \%$ of college students chose to accept it in their hearts, which shows that the overall patriotism of college students is relatively strong, but the percentage of those who can practice it is only $13 \%$. Indeed, 


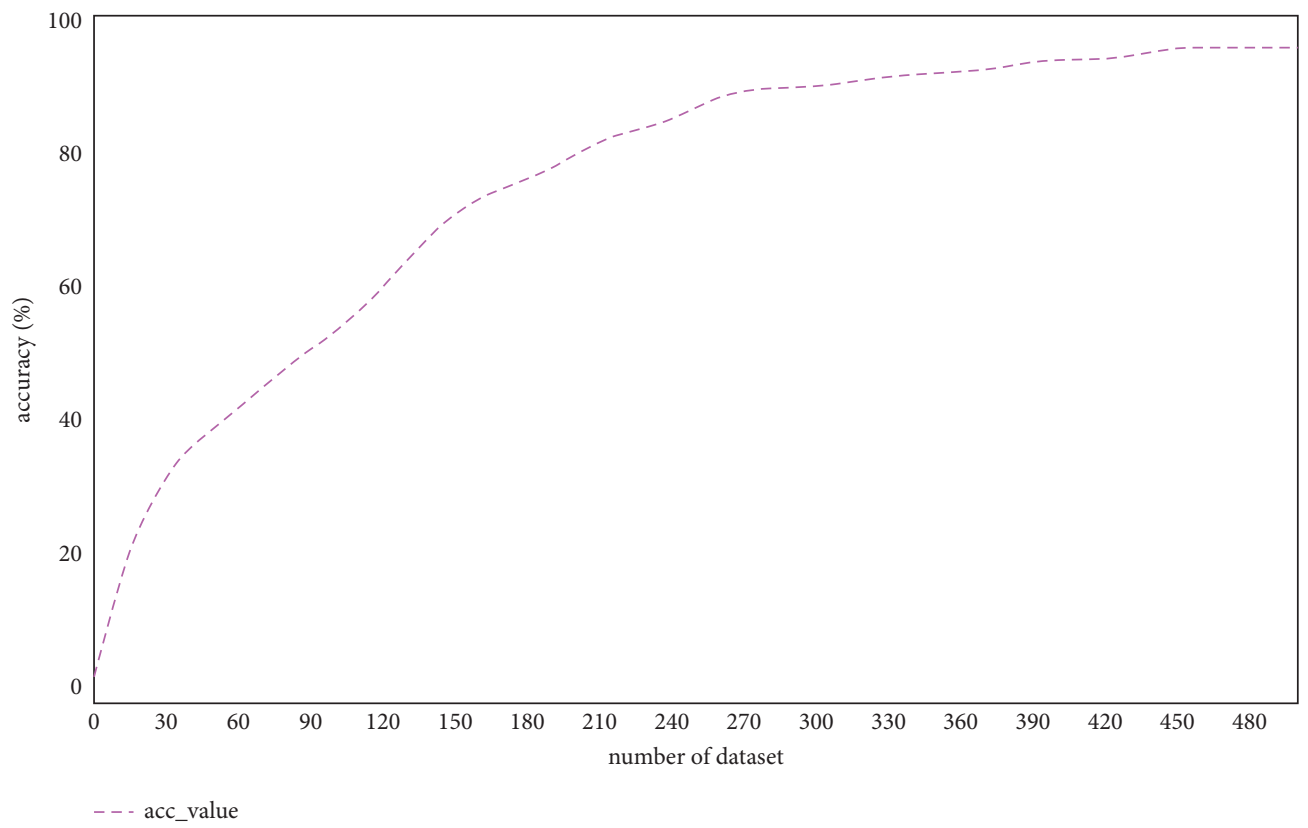

Figure 7: Data volume and accuracy trend graph.

TABle 4: C2 statistics table.

\begin{tabular}{lccccc}
\hline & Resist & Understand & Agree & Acceptance & Behavioral practices \\
\hline Number of people & 1 & 3 & 12 & 71 & 13 \\
Percentage & 1 & 3 & 12 & 71 & 13 \\
Number of model output & 1 & 2 & 11 & 72 & 14 \\
\hline
\end{tabular}

being in the peace era, there are not many opportunities provided for college students to go to the battlefield.

4.3.3. C3 Category Acceptance Status. From the data in Table 5, the total number of college students who chose "accept it in their hearts" and "practice it" is 77 , which is slightly lower than "serve the nation with loyalty and national integrity." However, the proportion of those who can set an example and put it into practice has increased to a certain extent. $23 \%$ of college students still chose "familiar with" or "below"; in particular, the proportion is higher among the junior students.

4.3.4. C4 Category Acceptance Status. From the data in Table 6, it is clear that the number of college students who accept "honesty, knowledge, humility, and love" is significantly lower than the first two, and the number of those who can put it into practice is also reduced accordingly. Some pessimists have argued that honesty is leaving our time and the world is full of frauds and lies.

4.3.5. C5 Category Acceptance Status. From the data in Table 7 , it can be seen that, among the above four statistics, college students accept the highest degree of human morality such as "family harmony and filial piety" and are able to put it into practice. As we know, most of the college students choose to buy gifts to express their filial respect to their parents and elders, which is a way to express their feelings but not enough.

4.3.6. C6 Category Acceptance Status. From the data in Table 8 and the comparison of college students' school performance, the author found that most of the students who chose to practice behavior ranked in the top third of their grade, which fully illustrates the importance of practice for improving college students' own ability. Most of the respondents who chose to accept it said that they agree with the expected benefits brought by the efforts, but they are always unable to devote themselves to their study or work due to various distracting factors, and their attention is very easily distracted, and they often end up in a tiger's head and stop halfway.

4.3.7. Graphical Representation. Figure 8 depicts a graphical depiction of the acceptance status of the $\mathrm{C} 2$ category. In this figure, the author has taken the number of persons who participated in the experimental work, their percentage, and the number of models output. This shows that just one person resisted, three people understood, twelve agreed, and seventy-one accepted it with thirteen behavioral practices. Furthermore, in the author's model output one resisted, two understood, eleven agreed, and seventy-two accepted behavior patterns. 
TABLE 5: C3 statistics table.

\begin{tabular}{lccccc}
\hline & Resist & Understand & Agree & Acceptance & Behavioral practices \\
\hline Number of people & 2 & 5 & 16 & 57 & 20 \\
Percentage & 2 & 5 & 16 & 57 & 20 \\
Number of model output & 2 & 5 & 15 & 56 & 22 \\
\hline
\end{tabular}

Table 6: C4 statistics table.

\begin{tabular}{lccccc}
\hline & Resist & Understand & Agree & Acceptance & Behavioral practices \\
\hline Number of people & 3 & 4 & 28 & 54 & 11 \\
Percentage & 3 & 4 & 28 & 54 & 11 \\
Number of model output & 2 & 3 & 26 & 56 & 13 \\
\hline
\end{tabular}

TABle 7: C5 statistics table.

\begin{tabular}{lccccc}
\hline & Resist & Understand & Agree & Acceptance & Behavioral practices \\
\hline Number of people & 1 & 2 & 7 & 45 & 45 \\
Percentage & 1 & 2 & 7 & 45 & 45 \\
Number of model output & 0 & 1 & 8 & 46 & 45 \\
\hline
\end{tabular}

TABle 8: C6 statistics table.

\begin{tabular}{lccccc}
\hline & Resist & Understand & Agree & Acceptance & Behavioral practices \\
\hline Number of people & 3 & 4 & 23 & 45 & 25 \\
Percentage & 3 & 4 & 23 & 45 & 25 \\
Number of model output & 2 & 3 & 21 & 47 & 27 \\
\hline
\end{tabular}

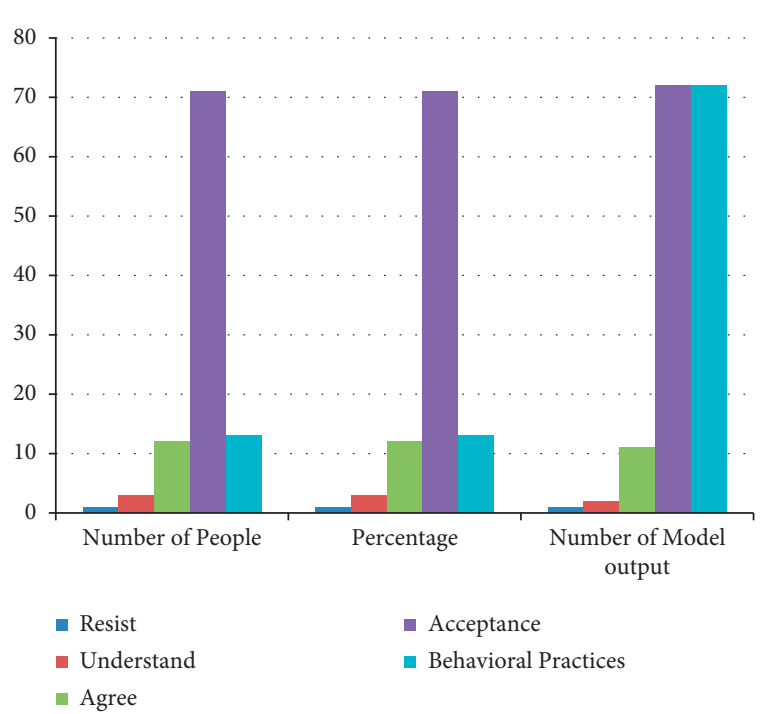

FIgURE 8: Representation of the acceptance status of the C2 category.

Figure 9 shows a graphical depiction of the acceptance status of the C3 category. Here the author has taken the number of persons who participated in the experimental work, their percentage, and the number of models output. This shows that just two people resisted, five people understood, sixteen agreed, and fifty-seven accepted it with twenty behavioral practices. Similarly, two percent resisted,

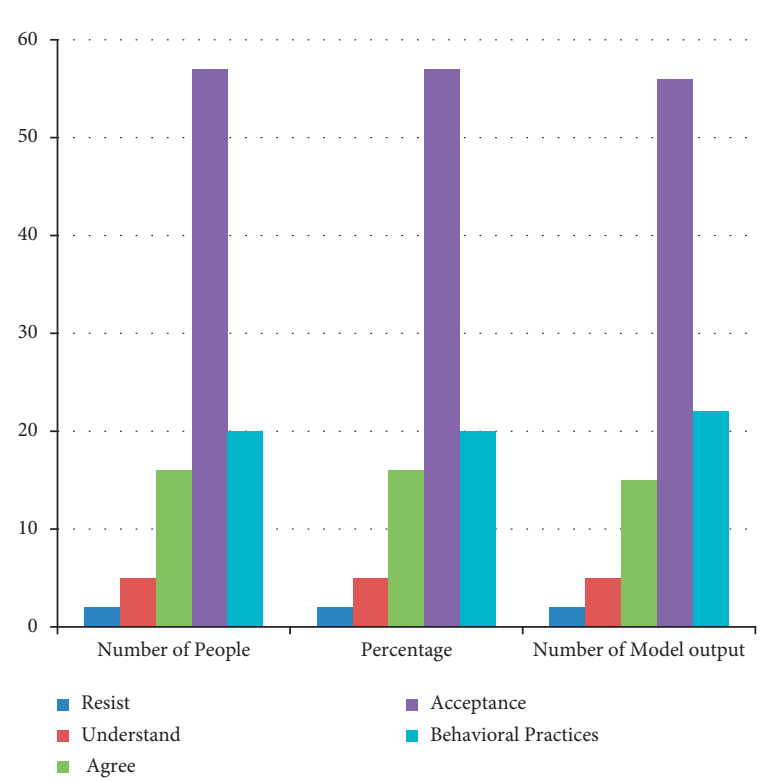

Figure 9: Representation of the acceptance status of the $\mathrm{C} 3$ category.

five percent understood, sixteen agreed, and fifty-seven accepted it with twenty behavioral practices. Furthermore, in the author's model output two resisted, five understood, fifteen agreed, and fifty-six accepted with twenty-two behavior patterns. 


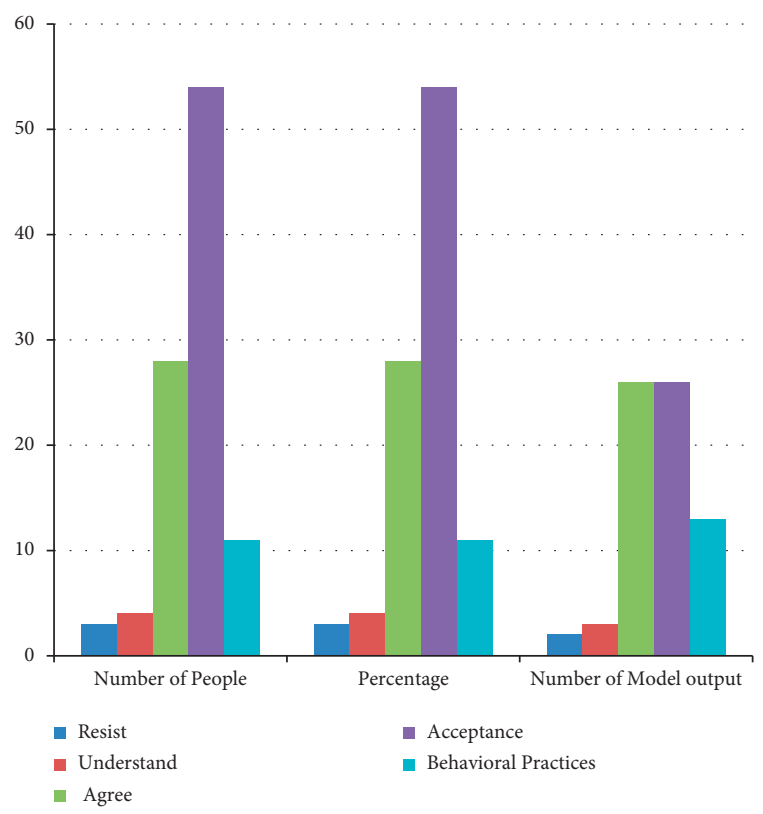

Figure 10: Representation of the acceptance status of the C3 category.

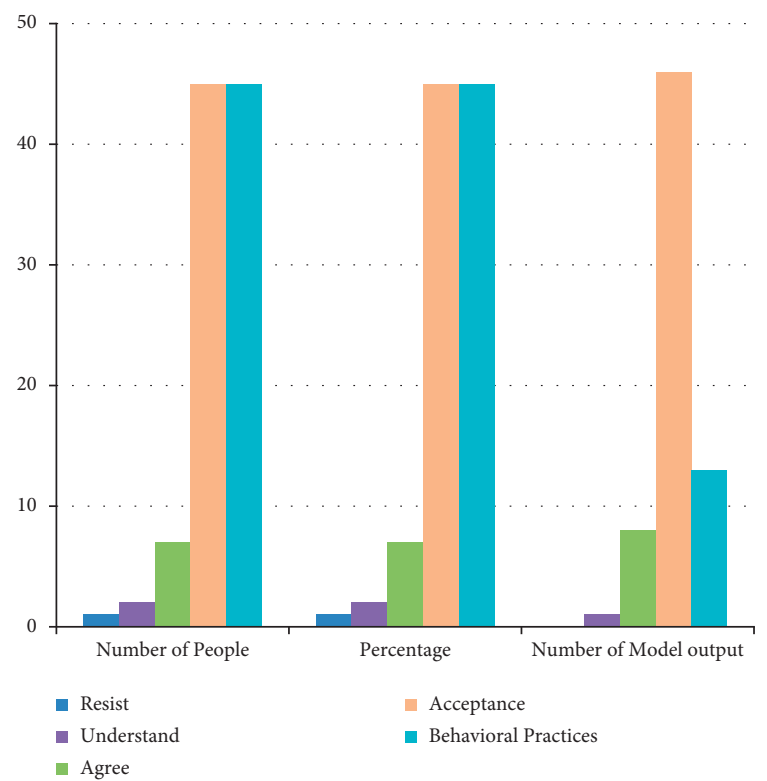

Figure 11: Representation of the acceptance status of the C5 category.

Figure 10 shows a graphical depiction of the acceptance status of the $\mathrm{C} 4$ category. Here, the author has taken the number of persons who participated in the experimental work, their percentage, and the number of models output. This shows that three people resisted, four understood, twenty-eight agreed, and fifty-four accepted it with eleven behavioral practices. Similarly, three percent resisted, four percent understood, twenty-eight agreed, and fifty-four accepted it with eleven behavioral practices. Furthermore, in the author's model output two resisted, three understood, twenty-six agreed, and fifty-six accepted with thirteen behavior patterns.

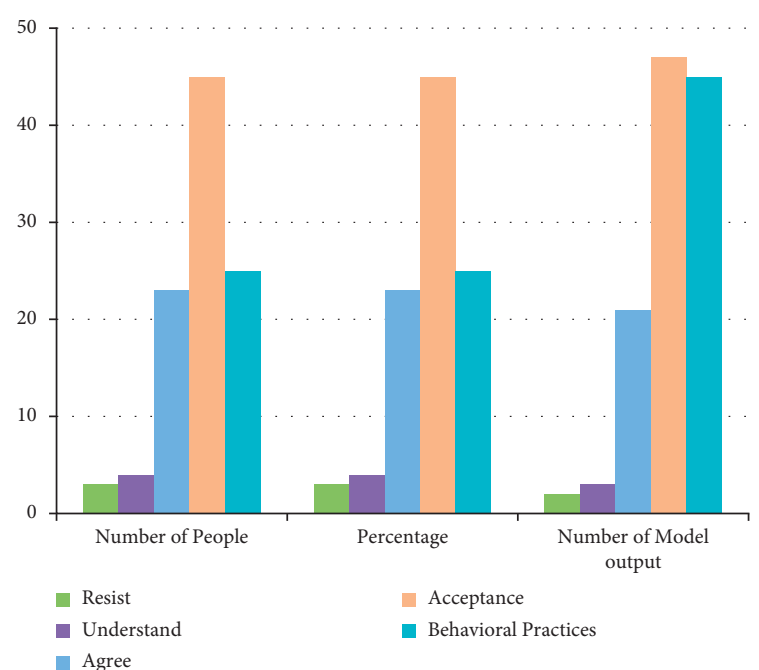

FIgURE 12: Representation of the acceptance status of the C6 category.

Figure 11 shows a graphical depiction of the acceptance status of the C5 category. Here the author has taken the number of persons who participated in the experimental work, their percentage, and the number of models output. This shows that only one person resisted, two understood, seven agreed, and forty-five accepted it with forty-five behavioral practices. Similarly, one percent resisted, two percent understood, seven agreed, and fifty-five accepted it with forty-five behavioral practices. Furthermore, in the author's model output zero resisted, one understood, eight agreed, and forty-six accepted with forty-five behavior practices.

Figure 12 shows a graphical depiction of the acceptance status of the C6 category. Here the author has taken the number of persons who participated in the experimental work, their percentage, and the number of models output. This shows that three people resisted, four understood, twenty-three agreed, and forty-five accepted it with twentyfive behavioral practices. Similarly, three percent resisted, three percent understood, twenty-three agreed, and fortyfive accepted it with twenty-five behavioral practices. Furthermore, in the author's model output two resisted, three understood, twenty-one agreed, and forty-seven accepted with twenty-seven behavior practices.

\section{Conclusion}

In this paper, the author conducted a study on the theme of college students' acceptance of Chinese excellent traditional moral culture, and the acceptance state here is not a broad discussion of acceptance in philosophy, but a separate analysis of the "state" of it, using various methods such as logical analysis, questionnaire survey, and mathematical statistics, based on fully absorbing the existing research results. To begin with, the adoption of good traditional Chinese moral culture among college students is a significant issue. To begin with, the adoption of good traditional moral culture by college students is important not only for the 
development and promotion of socialist core values but also for encouraging college students to become suitable socialist successors. Second, the author discovered that the main issues are a lack of awareness among college students, an imperfect education mechanism, and a lack of practical activities, which are caused by a variety of factors such as social evolution and cultural conflicts, school reform, and teacher quality, family moral education environment, and college students' ability to self-restraint. This paper proposes a model based on a convolutional neural network that predicts college students' acceptance status of Chinese excellent traditional moral culture based on their behaviors and preferences in social networks to address the problem that it is difficult to identify college students' acceptance status of Chinese excellent traditional moral culture. Experiments were done in the test set by assembling the dataset with actual questionnaires to establish the validity and accuracy of the proposed model, laying the groundwork for future instruction on Chinese superb traditional moral culture for college students' groups.

\section{Data Availability}

The datasets used during the current study are available from the corresponding author on reasonable request.

\section{Conflicts of Interest}

The author declares that he has no conflicts of interest.

\section{References}

[1] Y.-t. Zhu, "The birth of traditional Chinese ethics in west zhou (1046-771 BC)," A Panoramic History of Traditional Chinese Ethics, Springer, Singapore, 2021.

[2] G. Lin, "Research on the application of traditional Chinese medicine culture in the construction of teachers' ethics system in medical colleges-take Zhangzhou health vocational college as an example," Frontiers in Educational Research, vol. 3, no. 14, 2020.

[3] Y. Yan, "The drive for success and the ethics of the striving individual," Ordinary Ethics in China, Routledge, Milton Park, UK, pp. 263-291, 2020.

[4] G. Shan, "From intrinsic value to the emotion of wonder," Environmental Ethics, vol. 40, no. 1, pp. 81-91, 2018.

[5] Z. Yu, J. Pei, M. Zhu, J. Zhang, and J. Li, "Multi-attribute adaptive aggregation transformer for vehicle re-identification," Information Processing and Management, vol. 59, no. 2, Article ID 102868, 2022.

[6] J. Zhu, T. Stone, and M. Petrini, "The ethics of refusing to care for patients during the coronavirus pandemic: a Chinese perspective," Nursing Inquiry, vol. 28, no. 1, Article ID e12380, 2021.

[7] M. Wen, S. Zhang, and D. McGhee, "Utilizing the moral nobility of older Chinese women in governance: the uses of humility, empathy, and an ethics of care in moral clinics in Huzhou city," British Journal of Sociology, vol. 71, no. 2, pp. 300-313, 2020.

[8] Y. Lian-yun and P. Jing, "Construction of life-practice moral education based on traditional Chinese morality with life connotation," Frontiers of Education in China, vol. 1, no. 3, pp. 456-461, 2006.
[9] Y. Yan and P. Chen, "The influence of traditional Chinese culture on the University student's ecological moral education," Journal of Jiangsu Polytechnic University (Social Science Edition), vol. 1, 2010.

[10] L. Zhang, Y. Wang, M. Xiao, Q. Han, and J. Ding, "An ethical solution to the challenges in teaching anatomy with dissection in the Chinese culture," Anatomical Sciences Education, vol. 1, no. 2, pp. 56-59, 2008.

[11] D. Y. F. Ho, W. Xie, X. Liang, and L. Zeng, "Filial piety and traditional Chinese values: a study of high and mass cultures," PsyCh Journal, vol. 1, no. 1, pp. 40-55, 2012.

[12] H. L. Yang and W. P. Wu, "The effect of moral intensity on ethical decision making in accounting," Journal of Moral Education, vol. 38, no. 3, pp. 335-351, 2009.

[13] R. Hayhoe, "The evolution of modern Chinese educational institutions," Contemporary Chinese education, Routledge, Milton Park, UK, pp. 26-234, 2017.

[14] J. Wen, E. Thibeau-Sutre, M. Diaz-Melo et al., "Convolutional neural networks for classification of Alzheimer's disease: overview and reproducible evaluation," Medical Image Analysis, vol. 63, Article ID 101694, 2020.

[15] B. Chapaliuk, "Overview of the three-dimensional convolutional neural networks usage in medical computer-aided diagnosis systems," American Journal of Neural Networks and Applications, vol. 6, no. 2, pp. 22-28, 2020.

[16] A. Palvanov and Y. Cho, "VisNet: deep convolutional neural networks for forecasting atmospheric visibility," Sensors, vol. 19, no. 6, p. 1343, 2019.

[17] B. Park, H. Park, S. M. Lee, J. B. Seo, and N. Kim, "Lung segmentation on HRCT and volumetric CT for diffuse interstitial lung disease using deep convolutional neural networks," Journal of Digital Imaging, vol. 32, no. 6, pp. 1019-1026, 2019.

[18] M. Islam, G. Chen, and S. Jin, "An overview of neural network," American Journal of Neural Networks and Applications, vol. 5, no. 1, pp. 7-11, 2019.

[19] L. Lazebnik, "Convolutional neural network architectures: from LeNet to ResNet," Presentation, University of Illinois, accessed, vol. 20, 2018.

[20] K. He, X. Zhang, S. Ren, and S. Jian, "Deep residual learning for image recognition," in Proceedings of the IEEE Conference on Computer Vision and Pattern Recognition, pp. 770-778, Las Vegas, NV, USA, December, 2016.

[21] Z. Lu, P. Du, and J.-Y. Nie, "VGCN-BERT: augmenting BERT with graph embedding for text classification," Lecture Notes in Computer Science, vol. 12035, pp. 369-382, 2020.

[22] Z. J. Wang, R. Turko, O. Shaikh et al., "CNN explainer: learning convolutional neural networks with interactive visualization," IEEE Transactions on Visualization and Computer Graphics, vol. 27, no. 2, pp. 1396-1406, 2020.

[23] D. H. Hubel and T. N. Wiesel, "Receptive fields, binocular interaction and functional architecture in the cat's visual cortex," The Journal of Physiology, vol. 160, no. 1, pp. 106-154, 1962.

[24] Y. LeCun, B. E. Boser, J. S. Denker et al., "Handwritten digit recognition with a back-propagation network," in Advances in Neural Information Processing SystemsMorgan Kaufmann Publishers Inc., San Francisco, CA, USA, 1990. 